\title{
Behavior and Knowledge of Prescribing Antibiotics by Physicians in Dammam Medical Complex, in Dammam, Kingdom of Saudi Arabia in Summer 2014
}

\author{
Bahaa Eldin Fateha*, Hadeel Shihabi*, Nouf Albalawi*, Bashayer Alsulami, Marwa Alsunaidi, Norah Alhoti, \\ Rand Alharthi, Rawan Alkharraz, Suja Alzayer and Wejdan Almotairi
}

Department of Family \& Community Medicine, Arabian Gulf University, Bahrain

Submission: February 01, 2019; Published: February 22, 2019

*Corresponding author: Bahaa Eldin Fateha, Hadeel Shihabi \& Nouf Albalawi, Department of Family and Community Medicine, Arabian Gulf University, Bahrain

\begin{abstract}
This study was conducted in Dammam in Summer 2014. The aim of the study is to observe the knowledge and behaviour of Dammam medical complex physicians toward antibiotic drug prescription and raise the awareness about the danger of random prescription of antibiotics. The objectives of the study are: to know whether physicians order sensitivity test before prescribing antibiotic drugs or not, to determine the relevance of years of experience of the physician to prescription behaviour of antibiotic, to know what are the factors that influence the prescription of antibiotics. The study is a cross sectional study, it included 403 Physicians in Dammam medical complex. The key findings of the study were as follows,

a) physicians with longer years of experience do rely on many factors in prescribing antibiotics and they do most of the time order antibiotic sensitivity test,

b) physicians with longer years of experience tend to give special precautions for special cases,

c) physicians with longer years of experience are never affected by recommendations from medical representatives, also they don't put into consideration the side effects of a drug before prescribing antibiotics, but they do always explain the side effects for their patients, and they do follow them up to monitor the side effects,

d) physicians with longer years of experience are affected by the patient's visit time at Dammam Medical Complex. In short, physicians with longer years of experience are more careful about prescribing antibiotics than those with shorter years of experience.
\end{abstract}

Keywords: Behavior; knowledge of prescribing antibiotics; Prescribing antibiotics in dammam Medical Complex; Factors influence prescription of antibiotics

Abbreviation: MRSA: Methicillin-Resistant Staphylococcus Aureus; USA: United States of America; CDC: Centers for Disease Control; WHO: World Health Organization; DMC: Dammam Medical Complex

\section{Introduction}

Antibiotics are substances produced by or derived from microorganisms that destroy or inhibit the growth of other microorganisms. Antibiotics are used to treat infections caused by organisms that are sensitive to them, usually bacteria or fungi. On the other hand, antibiotics may alter the normal microbial content of the body (e.g. in the intestine, lungs, bladder) by destroying one or more groups of harmless beneficial organisms, which may result in infections (such as thrush in women) due to overgrowth of resistant organisms. These side effects are most likely to occur in broad-spectrum antibiotics (those active against a wide variety of organisms). Resistance may also develop in microorganisms being treated; for example, through incorrect dosage or over prescription. Antibiotics should not be used to treat minor infections, which will clear up unaided. Some antibiotics may cause allergic reactions [1]. Antibiotic resistance can be caused also by taking an antibiotic too often for a condition it cannot treat such as viral infections and the common cold or in the wrong doses. It can also be manifested by not finishing a course of antibiotics as prescribed (stopping the antibiotic before the infection is fully cleared from the body) [2]. A prominent example of the dangers of antibiotic resistance is the spread of methicillin-resistant Staphylococcus aureus 


\section{Juniper Online Journal of Public Health}

"MRSA" [3]. MRSA was once a concern only for hospitalized patients, but a newer form of MRSA is causing infections in healthy people in the community [4]. Infections that are caused by bacteria resistant to antibiotics are difficult to treat.

These infections need to be treated with more expensive toxic medications, or if left without treatment they will result in prolonged illnesses and can cause death. Unfortunately, antibiotic-resistance can develop quickly, and this is a global health concern. Moreover, antibiotic resistance threatens a return to pre-antibiotic era [3]. More than $50 \%$ of all medicines are prescribed, dispensed or sold inappropriately, and half of all patients fail to take medicines correctly. The overuse, underuse or misuse of medicines harms people and wastes resources. More than $50 \%$ of all countries do not implement basic policies to promote rational use of medicines. In developing countries, less than $40 \%$ of patients in the public sector and $30 \%$ in the private sector are treated according to clinical guidelines. A combination of health-care provider education and supervision, consumer education, and an adequate medicines supply is effective in improving the use of medicines, while any of these interventions alone has limited impact. There are some factors that contribute to the incorrect use of antibiotics such as: lack of skills and knowledge, inappropriate unethical promotion of medicines by pharmaceutical companies, profits from selling medicines, unrestricted availability of medicines, overworked health personnel, unaffordable medicines and lack of coordinated national pharmaceutical policy [5]. More than a dozen new classes of antibiotics were developed in the 1930s through the 1960s, but only two new classes have been developed since then. In a study of the top 15 pharmaceutical companies, only 1.6\% of drugs in development were antibiotics, none of which were from novel classes and leaving need unmet for multi-resistant Gram-negative infections. Thus, With the loss of effect of existing antibiotic and slow development of new antibiotics the World Health Organization requested a massive effort to prevent emerging antibiotic resistance, and shortly thereafter presented a global strategy for the containment of antimicrobial resistance, calling for a multidisciplinary and coordinated approach. However, sufficient financial and human resources to implement the strategy were never provided [6].

\section{Literature Review}

A study by N.M Abdulla [7] aimed at assessing the underlying causes of antibiotics resistance in Saudi Arabia. About 50 patients were randomly selected who are proved to have Staphylococcus antibiotic resistance in Assir hospital microbiology laboratory. Their study found that about $64 \%$ of patients used to exaggerate their symptoms in order to receive a prescription of antibiotic drugs. About $64 \%$ of patients used to have the antibiotic dosage described by treating physicians and $20 \%$ by pharmacists. About 35 patients (70\%) have received description of the drug side effects [7]. Also, a study by Abobotain et al. [8]. about sociodemographic determinants of antibiotic misuse in children, a survey from the central region of Saudi Arabia showed that from 610 parents (60.5\% mothers and $39.5 \%$ fathers), $11.6 \%$ reported self-prescription. Responding parents differed by age, education, number of children, and profession. Overall, parents responded that antibiotics are required in children with runny nose or cough or sore throat or fever (50\%); to reduce symptom severity and duration (57.7\%), are effective against viruses (68.6\%), can be stopped on clinical improvement (28.7\%), and it is used by another family member can be used in children (20.1\%). A study by Bilal et al. [9] about endemic nosocomial infections and misuse of antibiotics in a maternity hospital in Saudi Arabia, showed that patients admitted during a 6-month period to a maternity hospital in Saudi Arabia were studied for nosocomial infections and misuse of antibiotics. Patient history and diagnosis on admission and subsequent clinical and laboratory data were analysed $[8,9]$.

This study found that over $90 \%$ of the bacterial isolates were multidrug-resistant. About $24 \%$ of patients received single or multiple antibiotics; $57.2 \%$ were misused. The minimal hospital cost estimate for both nosocomial infections and misused antibiotics was US \$318,705 [9]. Between 2001 and 2005, there was an estimate that there were $14,738,000$ incidents of moderate to severe antibiotic adverse drug reactions in China yearly, and that 150,000 patients died (Xiao et al.) [10]. Other studies have focused on antibiotic prescriptions for specific groups like Medicare patients in USA. Doctors and other health care providers prescribed 258 million courses of antibiotics in 2010, for a population of 309 million, which translates to 833 antibiotic prescriptions for every 1,000 people, on average (2013). During the swine flu pandemic of 2009 and 2010, a lot of doctors specially in USA in the south states prescribed antibiotics and antiviral at the same time because of the chances of developing flu-related pneumonia and to prevent all forms of deadly complications (2013) [11]. Another study regarding a survey of patterns, attitudes, and beliefs of house staff physicians concerning antibiotic use in Khartoum state hospitals between Jan - Feb 2010. About the basis of prescribing antibiotics to the Patient, $12.2 \%$ of physicians prescribe antibiotics according to their personal experience, $11 \%$ according to culture sensitivity. On prescribing antibiotics $95.6 \%$ explain indications and usages of antibiotics and also expectation from antibiotics. $87.3 \%$ of prescribers explain side effects to their patients and $78.5 \%$ refer back to patient to follow side effects after prescribing antibiotics. Reasons and situations of prescribing antibiotics as prophylaxis in their specialty is to reduce post- operative wound infection was the main reason for antibiotics prescribing (90.5\%) [12]. A study examined the knowledge and practice of antibiotic use among students in a university in south western Nigeria revealed that a larger proportion of the respondents $(72.4 \%)$ use the same antibiotics as previously prescribed by their doctor to treat an infection. The most reasons for using antibiotics showed that $32.3 \%$ and $17.7 \%$ of respondents used antibiotics for common cold and sore throat respectively [13]. A study was done to assess physicians' knowledge and perception regarding antibiotic resistance and current antibiotic practices at Khartoum 
State-Sudan between October 2011 and March 2012 showed $(51.7 \%)$ of respondents considered antibiotic resistance was a major problem when prescribing antibiotics. $39.1 \%$ agreed that antimicrobials were overused in their institutions [14]. A study designed by the CDC revolved around the misuse of antibiotics proved the most common types of infections for which hospital clinicians wrote antibiotic prescriptions were lung infections (22\%), urinary tract infections (14\%), and suspected infections caused by drug-resistant Staphylococcus bacteria, such as MRSA (17\%). About 1 out of 3 times, prescribing practices to treat urinary tract infections and prescriptions for the critical and common drug vancomycin included a potential error-given without proper testing or evaluation or given for too long [2].

A study by A Kotwani et al. [15] (26 July 2010) revealed that generally, in public primary health care facilities, microbiology lab facilities are not available and most of the patients who visit these facilities cannot afford the tests from private labs. So, doctors relied mainly on their clinical acumen. Also, newer antibiotics were used on the recommendations of medical representatives who present the doctors with skewed studies in favor of the antibiotic they want to market. As one doctor (GP11) elaborated: "The company representative gives only the good points of the products. He forces doctors to write the latest antibiotics. In addition, doctors from the private sector revealed that many in their profession prescribe those brands of antibiotics that provide them more profit or prescribe antibiotics even when they are not required to increase their profits. This is probably the reason for high consumption of newer and expensive brands of antibiotics. Moreover, doctors in the public sector said that they do not have enough time for each patient who comes to them to study a patient's case history, do a proper physical examination or educate them. This point is clearly reflected in the following statement of a public sector doctor (GP 2): 'Frankly speaking we have to move patients fast and we can't really argue so much. We have only two minutes per patient...' The public sector doctor points out that due to lack of time, they cannot educate a patient that his condition does not require an antibiotic as it will require an elaborate discussion and probably an argument. Therefore, to save time, doctors simply prescribe antibiotics without looking at the rationality of prescribing it [15].

\section{Study Aim and Objectives}

\section{Aim}

To observe the knowledge and behavior of Dammam medical complex physicians toward antibiotic drug prescription and raise the awareness about the danger of random prescription of antibiotics.

\section{Objectives}

a) To know whether physicians order sensitivity test before prescribing antibiotic drugs or not. b) To determine the relevance of years of experience of the physician to prescription behavior of antibiotics.

c) To know what are the factors that influence the prescription of antibiotics.

\section{Materials and Methods}

\section{Type of study}

Cross sectional study

\section{Data sources/collection}

Physicians in Dammam medical complex. All physicians in the hospital were included in the study.

\section{Study population/study sample}

Study population: The study population included all physicians in the hospital (549 physicians). All of them received the study questionnaire, and there were 403 respondents, Distribution of participants in the research was by gender, age, years of experience, specialization and nationality (Table 1).

Table 1: Distribution of participants in the research by gender, age, years of experience, specialization and nationality.

\begin{tabular}{|c|c|c|}
\hline & (No of Physician) $\mathbf{n = 4 0 3}$ & $\%$ \\
\hline Mender & & \\
\hline Female & 244 & 60.5 \\
\hline Nationality & 146 & 36.2 \\
\hline Saudi & 283 & 70.2 \\
\hline Non-Saudi & 81 & 20.1 \\
\hline Age Group & & \\
\hline Between 20 - 30 & 158 & 39.1 \\
\hline Between 31 - 40 & 123 & 30.6 \\
\hline Between 41 - 50 & 67 & 16.5 \\
\hline Between 51 - 60 & 30 & 7.3 \\
\hline Above 60 & 1 & 0.2 \\
\hline Years of Experience & & \\
\hline Below 5 years & 159 & 39.1 \\
\hline Between 5 - 9 years & 69 & 17.1 \\
\hline Between 10-19 years & 81 & 20.1 \\
\hline Between 20 - 29 years & 41 & 10 \\
\hline Above 30 years & 9 & 2 \\
\hline Specialization & & \\
\hline Resident \& Interns & 76.3 & \\
\hline Specialist & & \\
\hline Consultant & & \\
\hline
\end{tabular}

Study instrument/Date collection: Written Questionnaire was designed by the researchers. The questionnaire was written in English. (Attached at the end) and was distributed upon all physicians. 


\section{Ethical consideration}

a) Permission was obtained from the chairman of family and community medicine department to conduct the study.

b) Permission was obtained from the administrator of Dammam medical complex to conduct the study. c) Confidentiality of the respondents has been assured.

\section{Statistical analysis}

Statistical analysis was done based on descriptive statistics, using SPSS version21.

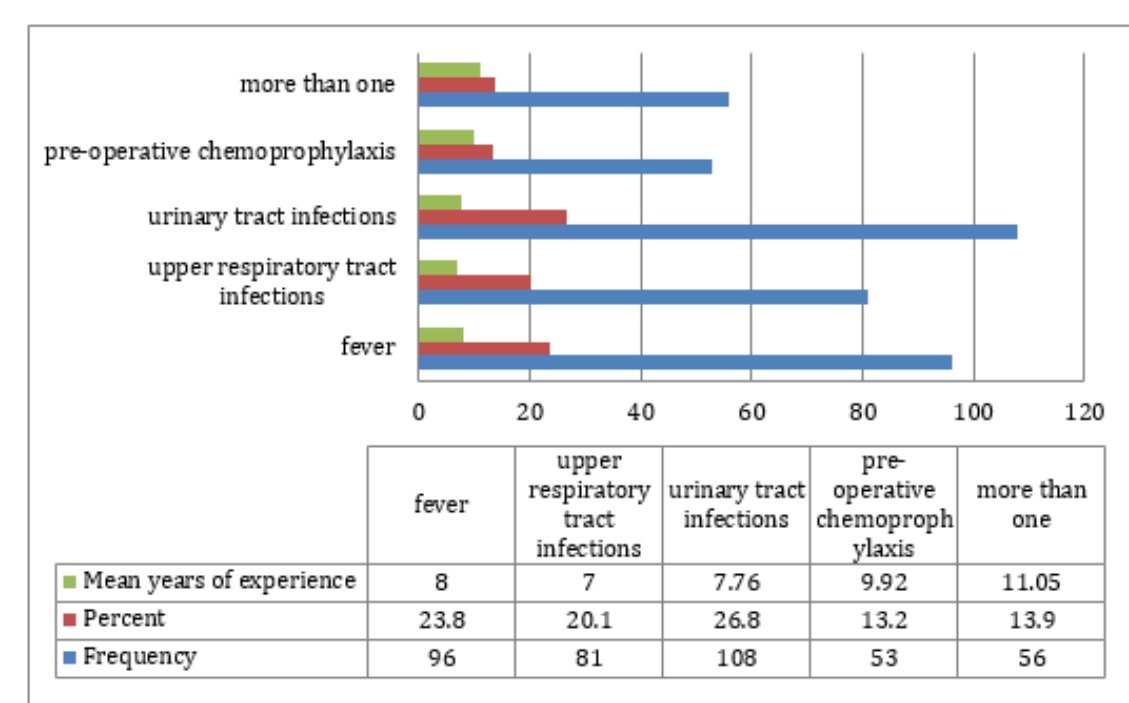

Figure 1: Relationship between years of experience and ordering antibiotic sensitivity test.

Table 2: Relevance of years of experience to factors influencing the prescription behaviour of antibiotics.

\begin{tabular}{|c|c|c|c|c|}
\hline The Factor & & Number & Percentage & Mean Years of Experience \\
\hline \multirow{4}{*}{ Availability of Antibiotics } & Always & 132 & $32.80 \%$ & 8.936 \\
\hline & Sometimes & 225 & $55.80 \%$ & 8.024 \\
\hline & Never & 46 & $11.40 \%$ & 9.511 \\
\hline & Missing & 0 & & \\
\hline \multirow{4}{*}{$\begin{array}{l}\text { Recommendations from Other Medical } \\
\text { Representatives }\end{array}$} & Always & 119 & $29.50 \%$ & 7.121 \\
\hline & Sometimes & 188 & $46.70 \%$ & 7.168 \\
\hline & Never & 96 & $23.80 \%$ & 12.402 \\
\hline & Missing & 0 & & \\
\hline \multirow{4}{*}{ Patient Visit Time } & Always & 101 & $25.10 \%$ & 9.834 \\
\hline & Sometimes & 256 & $63.50 \%$ & 7.844 \\
\hline & Never & 42 & $10.40 \%$ & 8.945 \\
\hline & Missing & 4 & $1 \%$ & \\
\hline \multirow{5}{*}{ Patient Satisfaction } & Always & 160 & $39.70 \%$ & 8.509 \\
\hline & Sometimes & 164 & $40.70 \%$ & 8.775 \\
\hline & Never & 76 & $18.90 \%$ & 7.962 \\
\hline & Other & 2 & $0.50 \%$ & 10 \\
\hline & Missing & 1 & $0.20 \%$ & \\
\hline \multirow{5}{*}{ Cost } & Always & 82 & $20.30 \%$ & 7.816 \\
\hline & Sometimes & 173 & $42.90 \%$ & 8.679 \\
\hline & Never & 146 & $36.20 \%$ & 8.855 \\
\hline & Other & 1 & $0.20 \%$ & \\
\hline & Missing & 1 & $0.20 \%$ & \\
\hline
\end{tabular}


Juniper Online Journal of Public Health

Side Effects

\begin{tabular}{|c|c|c|c|}
\hline Always & 281 & $69.70 \%$ & 9.331 \\
\hline Sometimes & 118 & $29.30 \%$ & 6.57 \\
\hline Never & 3 & $0.70 \%$ & 10.5 \\
\hline Missing & 1 & $0.20 \%$ & \\
\hline
\end{tabular}

\section{Results}

The key findings of the study were as follows,

a) physicians with longer years of experience do rely on

many factors in prescribing antibiotics and they do most of the time order antibiotic sensitivity test, (Figure 1) (Table 2).

b) physicians with longer years of experience tend to give special precautions for special cases, (Figure 2).

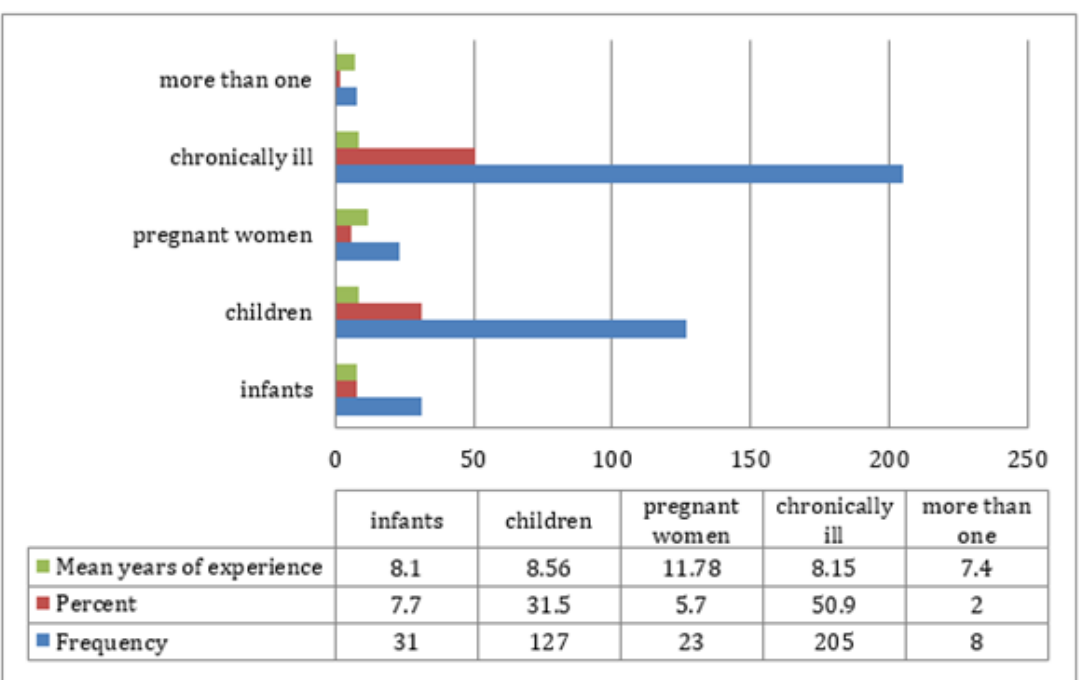

Figure 2: Relationship between years of experience and prescribing antibiotics to special medical cases.

c) physicians with longer years of experience are never affected by recommendations from medical representatives, also they don't put into consideration the side effects of antibiotics before prescribing them, but they do always explain the side effects for their patients, and they do follow them up to monitor the side effects (Figure $3 \& 4$ ).

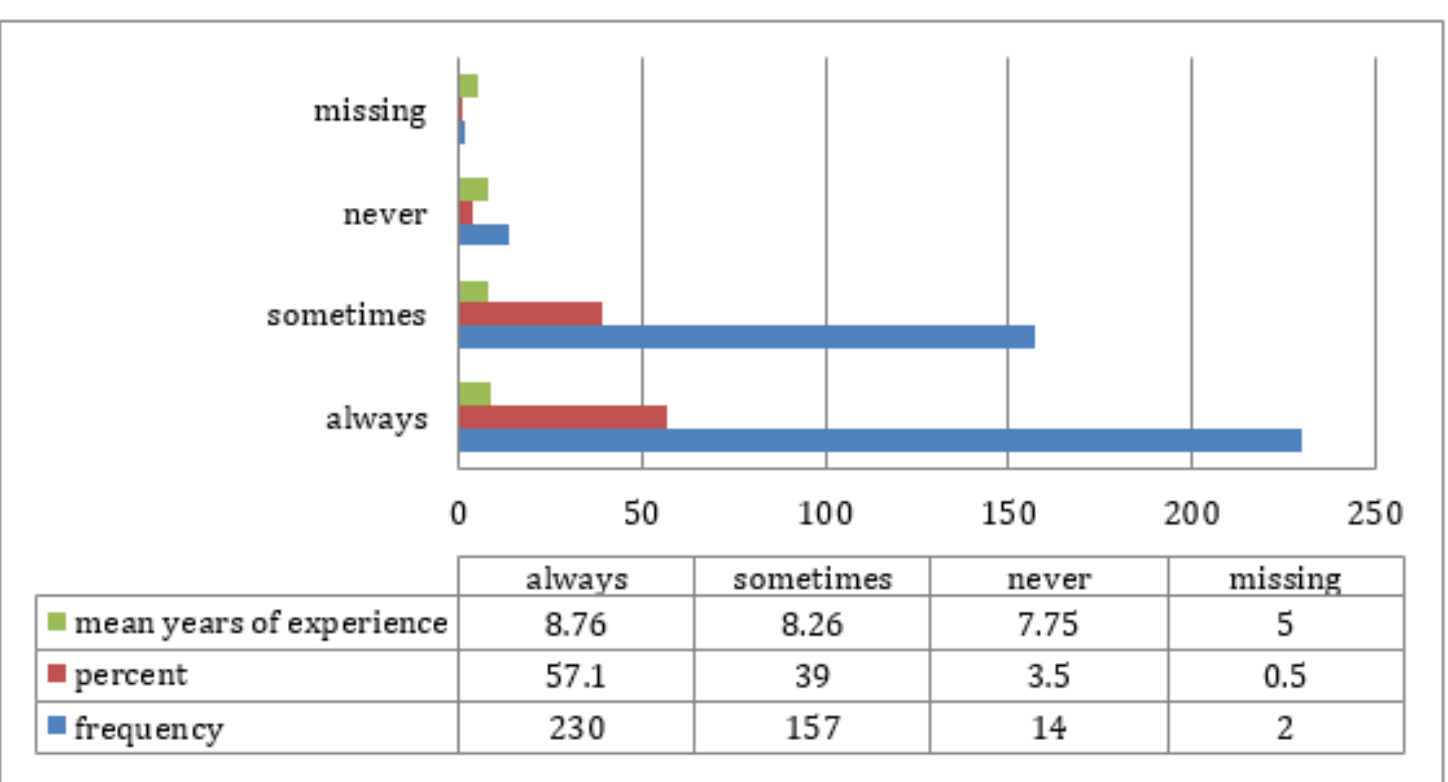

Figure 3: Relationship between years of experience and explaining the side effects of antibiotics to patients. 


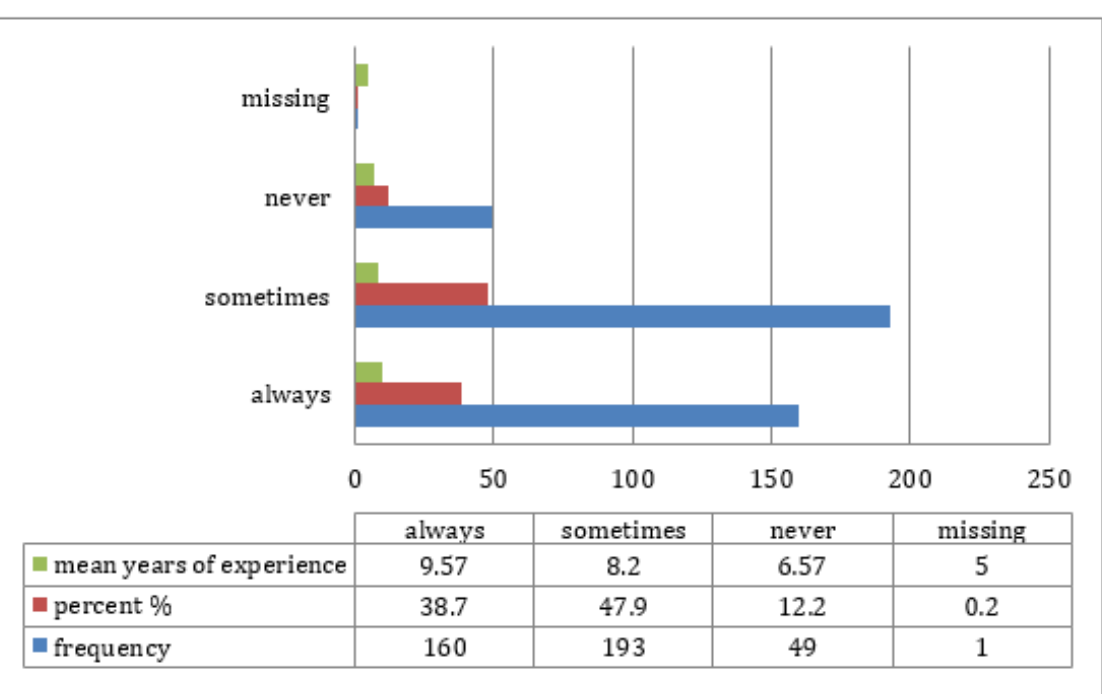

Figure 4: Relationship between years of experience and referring back the patient to follow up the side effects of the antibiotic.

d) physicians with longer years of experience are affected by the patient's visit time at Dammam Medical Complex. 403 Physicians were included in the study. Data analysis shows that $60.5 \%$ of physicians in the study were males and $36.2 \%$ were females. 215 of the total number of physicians were doctors under training (residents, interns), 71 specialist and 76 consultants. Table 1 illustrates the distribution of participants in the research by gender, age, years of experience, specialization and nationality.

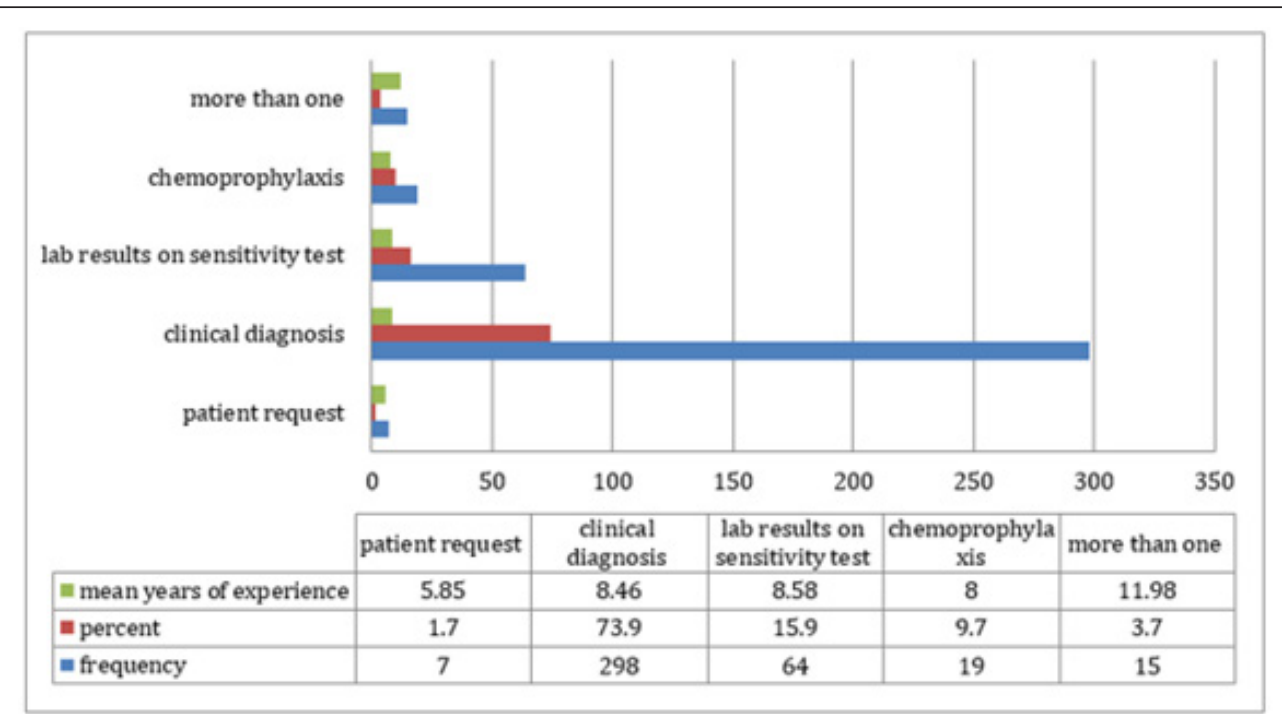

Figure 5: Relationship between years of experience and most common indication of prescribing antibiotics.

\section{Discussion}

Results showed that physicians with longer years of experience are more careful about prescribing antibiotics than those with shorter years of experience and this was expected because one of the factors that contribute to the incorrect use of antibiotics is lack of skills and knowledge and this agrees with WHO fact sheet [5]. One of the major findings is that physicians with longer years of experience do mostly order antibiotic sensitivity test and they do rely on other factors before prescribing antibiotics. On the other hand, physicians with shorter years of expe- rience rely on patients request in prescribing antibiotics (Figure $1 \& 5)$. This indicated that physicians with longer years of experience are more careful in prescribing antibiotics, as resistance to antibiotics can develop quickly and this goes in line with WHO recommendation [3]. Patients' request for antibiotics is not a good reason for prescribing antibiotics because some patients tend to exaggerate their symptoms in order to be prescribed antibiotics as it was shown in a study done by N.M. Abdalla [7]. Another important finding is that physicians with shorter years of experience do prescribe antibiotics for upper respiratory tract 
infections (Figure 6). Prescribing antibiotics for viral infections in one of the main aspects of improper usage of antibiotics and this goes in line of what has been shown in Chaudhary et.al. [2] study. Also, one of the findings in the study is that in special cases such as childhood, infancy, pregnancy, and chronic illness physicians with longer years of experience do give special precautions to patients with those cases which indicated the devotion of experienced physicians at DMC to give the important precautions to those special cases when prescribing the suitable antibiotics, and they reported that mostly they do prescribe antibiotics for pregnant women and this is an important finding and has a special significance as this requires extensive experience (Figure 2 \& 7). Another finding in the study is that physicians with less years of experience tend to change the antibiotic in case the pa- tient didn't show any improvement, while those with more years of experience chose more than one answer in the questionnaire as more than one approach can be made in order to achieve the best results (Figure 8). Moreover, physicians with more years of experience do always explain the side effects of antibiotics and they do refer their patients back to follow up the side effects. On the other hand, physicians with less years of experience don't explain the side effects of antibiotics; also, they never refer back their patients for following up the side effects. This explains that physicians with more years of experience agree to the importance of explaining the side effects to the patients as this help in compliance of the patient and sometimes the tolerance of the side effects (Figure 7-9).

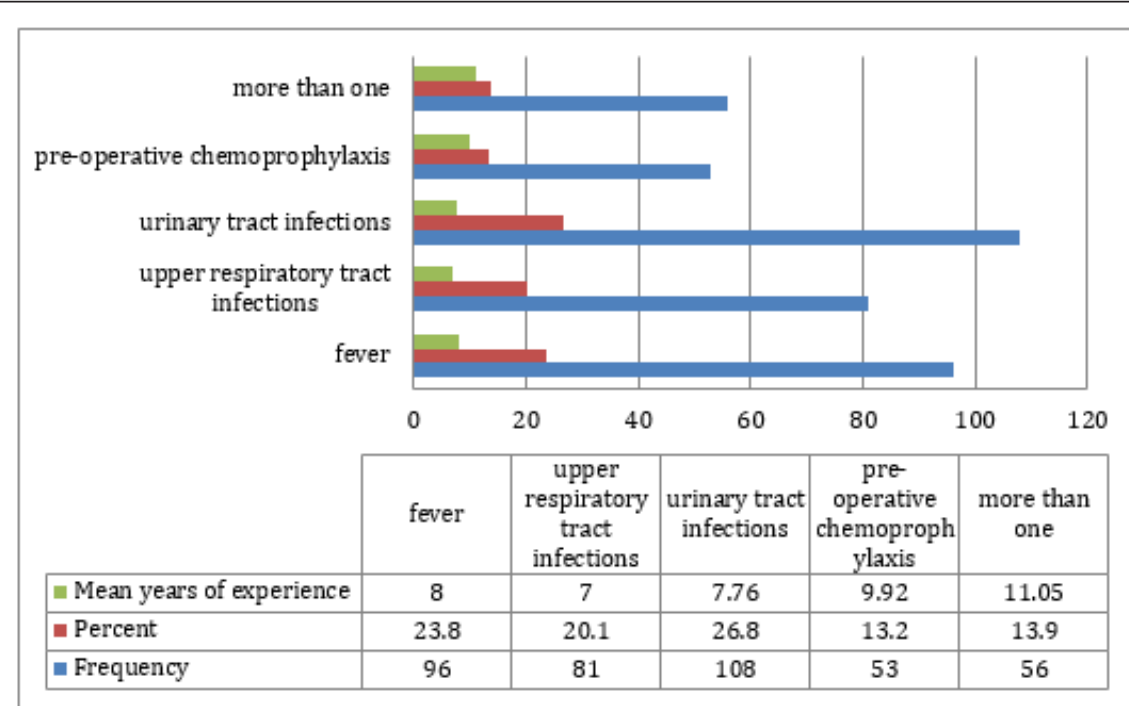

Figure 6: Relationship between years of experience and cases to which antibiotics are prescribed the most.

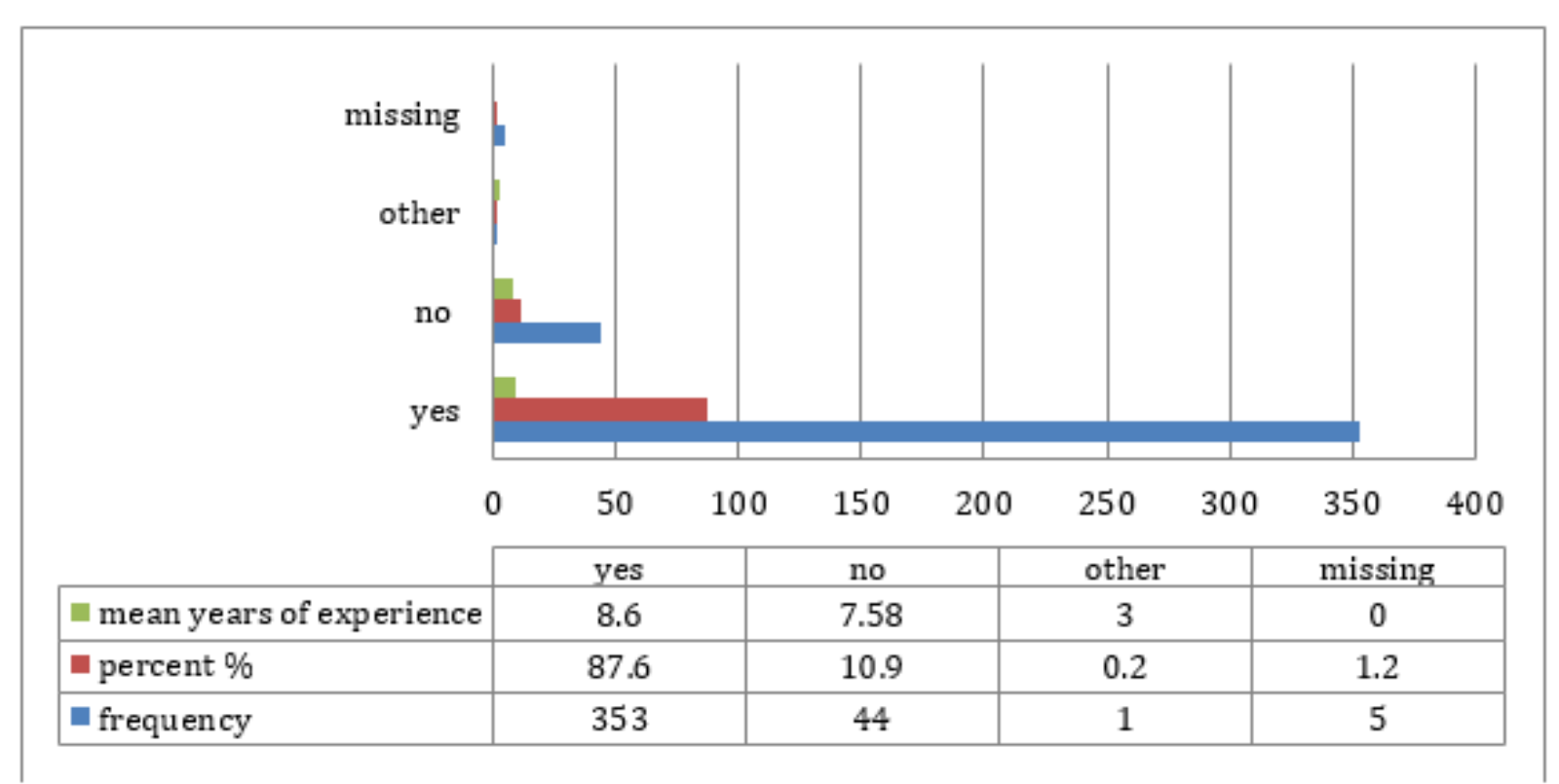

Figure 7: Relationship between years of experience and giving special precautions to special cases. 


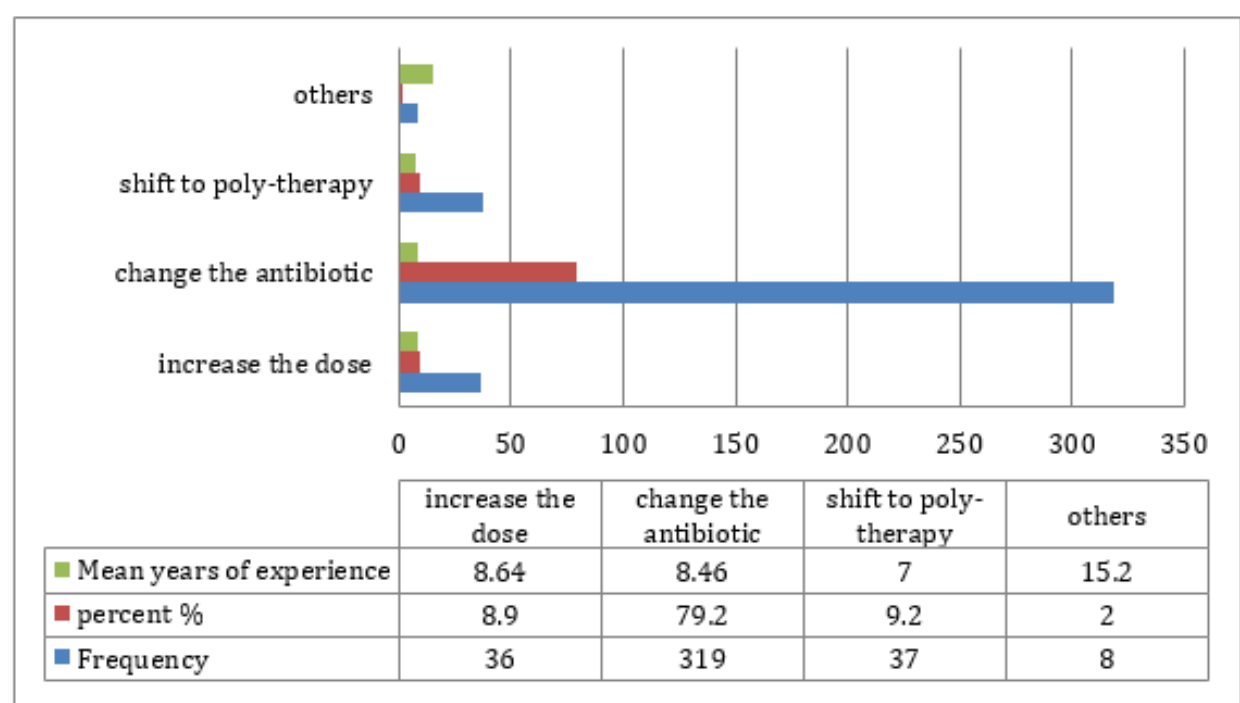

Figure 8: Relationship between years of experience and the action that is made in case of no response to antibiotic.

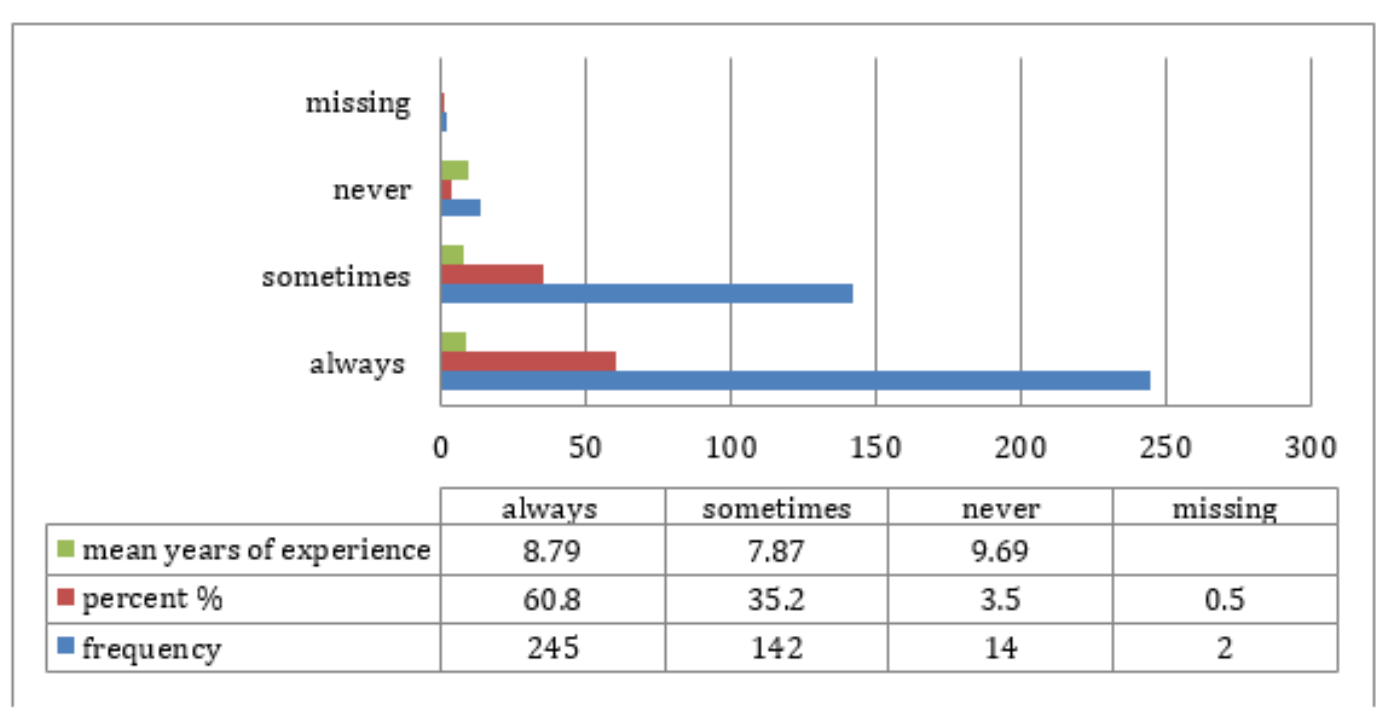

Figure 9: Relationship between years of experience and explaining the usage of antibiotics to patients.

There are many factors that influence the prescription of antibiotics and one of them is recommendation from medical representatives. Usually, medical representatives emphasize the advantages of a drug as it was shown in A Kotwani etal. [15] study. Fortunately, the results show that physicians with longer years of experience are never affected by recommendations from medical representatives (Table 2). Another factor that might influence the prescription of antibiotics is the side effects of a drug. Unfortunately, an expected finding was revealed in the results, physicians with longer years of experience are not satisfied with the current patient's visit time in this institution, as this factor can affect choosing the right antibiotic for the right patient (Table 2). That was expected because DMC is a public hospital and a lot of patients from Dammam and other nearby cities do seek medical care from this hospital and this agrees with A Kotwani et al. [15] study.

\section{Conclusion}

In this research, we were able to study the behavior of the prescribing the antibiotics and its relevance to the years of experience of the physicians in DMC. The significance of which, gave a clear idea about how physicians who were more experienced always order antibiotic sensitivity test and depend on more than one indication for prescribing antibiotics (clinical diagnosis, lab results on sensitivity test...). $21 \%$ of physicians in DMC reported that they always order antibiotic sensitivity test, while 58.6 reported that they sometimes do order antibiotic sensitivity test and $19.6 \%$ reported that they never order. This finding can raise awareness about the need to increase the number of physicians that always order antibiotic sensitivity test because of its great importance. Furthermore, we were able to study some of the factors that influence the prescription of 
antibiotics (availability of drugs, recommendation from other medical representatives, patient's visit time....) which was important as this could help in identifying the main influential factors that could be changed for better to achieve better prescription of antibiotics.

\section{Acknowledgement}

The authors would like to thank Dammam medical complex physicians who kindly devoted their time to participate. We would like to thank the Department of Family and Community Medicine, College of Medicine and Medical Sciences at Arabian Gulf University to assist us in conducting the research.

\section{References}

1. Elizabeth Martin (2003) Oxford concise medical dictionary. ( $7^{\text {th }}$ edn), University in Oxford, England, p. 39.

2. RR Chaudhury, A Kotwani (2012) Antibiotic overuse and increasing antibiotic resistance - time to react. South Asian Media Briefing

3. Fact sheet $\mathrm{N}^{\circ} 194$, WHO (2014) Antimicrobial resistance. WHO media centre.

4. Staff Mayo clinic (2012) Antibiotics: Misuse puts you and others at risk. Mayo Clinic, Patient care and health info.

5. Fact sheet $\mathrm{N}^{\circ} 338$, WHO (May 2010) Medicines: rational use of medicines. WHO media center.

6. Cars O, Högberg LD, Murray O, Nordberg O, Sivaraman S, et_al. (2008) Meeting the challenge of antibiotic resistance. BMJ.

7. Abdalla NM (2011) Study on Antimicrobial Resistant in Saudi Arabia. Med Well journals.

8. Abobotain AH, Sheerah HA, Alotaibi FN, Joury AU, Mishiddi RA, et al. (2013) Socio-demographic determinants of antibiotic misuse in children, A survey from the central region of Saudi Arabia. SMJ.

9. NE Bilal, M Gedebou, S Alghamdi (2002) Endemic nosocomial infections and misuse of antibiotics in a maternity hospital in Saudi Arabia.

10. Hamilton-Miller JMT (1984) Use and abuse of antibiotics. Europe PupMed Central.

11. Stobbe M (2013) Study shows overuse of antibiotics. USA Today.

12. Zaki AM, Kheder SI (2012) A survey of patterns, attitudes, and beliefs of house staff physicians concerning antibiotic use in Khartoum state hospitals. Elixir International Journal

13. Abimbola IO (1996) Knowledge and practices in the use of antibiotics among a group of Nigerian university students. International journal of infection control.

14. Kheder SI (2011) Physicians' Knowledge and Perception of Antimicrobial Resistance: A Survey in Khartoum State Hospital Settings. Science domain international.

15. Kotwani A, Wattal C, Katewa S, Joshic PC, Holloway K (2010) Factors influencing primary care physicians to prescribe antibiotics in Delhi India. Oxford Journals, USA.

\section{Personal data:}

1. Age:

2. Gender:

a) Female b) Male

3. Nationality:

4. Years of experience:

5. Years of working at Dammam Medical Complex:

6. Specialization:

a. Resident

b. Specialist

c. Consultant

\section{Questions:}

1. Q1: What is your most common indication for prescribing antibiotics?

a. Patient request

b. Clinical diagnosis

c. Lab result depending on sensitivity test

d. Chemoprophylaxis

2. Q2: Do you order an antibiotic sensitivity test before prescribing antibiotics to the patient?

a. Always.

b. Sometimes.

c. Never

3. Q3: Of the following, in which cases do you prescribe antibiotics the most?

a. Fever

b. Upper respiratory tract infections

c. Urinary tract infections

d. Pre- operative chemoprophylaxis

e. Post-operative chemoprophylaxis

f. Others: (Specify)

*Questions 4+5 are related

4. Q4: Of the following patients, to whom you prescribe antibiotics the most?

a. Infants.

b. Children

c. Pregnant women.

d. Chronically ill.

5. Q5: Do you give special precautions to those patients?

a. Yes.

b. No.

6. Q6: In case of no response to the antibiotic, what would you do?

a. Increase the dose.

b. Change the antibiotic. 
c. Shift to poly-therapy.

7. Q7: Do you explain the indications and the usages of antibiotic prescribed to the patient?

a. Always.

b. Sometimes.

c. Never.

8. Q8: Do you take the side effects into consideration when prescribing the suitable antibiotic for the patient?

a. Always.

b. Sometimes.

c. Never.

9. Q9: Do you explain clearly the important side effects of the antibiotic prescribed?
a. Always.
b. Sometimes.
c. Never.

10. Q10: Do you refer back the patient to follow up the side effects of the antibiotic prescribed?
a. Always.
b. Sometimes.
c. Never.

11. Q11: Does the availability of the antibiotic affect your decision of prescribing?
a. Always.
b. Sometimes.
c. Never.

12. Q12: Is your choice of antibiotic influenced by recommendations from medical representatives?
a. Always.
b. Sometimes.
c. Never.
*Questions $13+14$ are related

13. Q13: Do you think patient visit time in this institution is sufficient for choosing the right antibiotic for the patient?
a. Always.
b. Sometimes.
c. Never.

14. Q14: Do you think patient visit time in this institution influence your prescription of antibiotics?
a. Yes.
b. No.

15. Q15: Do you put into consideration patient satisfaction when prescribing antibiotics?
a. Always.
b. Sometimes.
c. Never.

16. Q16: Do you take the cost of the antibiotic into consideration before prescribing?
a. Always.
b. Sometimes.
c. Never. 